\title{
ORIENTATION STRATEGIES FOR AERIAL OBLIQUE IMAGES
}

\author{
A. Wiedemann, J. Moré
}

BSF Swissphoto, Mittelstr. 7, D-12529 Schönefeld, Germany - (albert.wiedemann, john.more)@bsf-swissphoto.com

\author{
Commission I, WG I/3
}

KEY WORDS: Aerial, Image, Aspects, Oblique, Orientation, Registration, Triangulation

\begin{abstract}
:
Oblique aerial images become more and more distributed to fill the gap between vertical aerial images and mobile mapping systems. Different systems are on the market. For some applications, like texture mapping, precise orientation data are required. One point is the stable interior orientation, which can be achieved by stable camera systems, the other a precise exterior orientation. A sufficient exterior orientation can be achieved by a large effort in direct sensor orientation, whereas minor errors in the angles have a larger effect than in vertical imagery. The more appropriate approach is by determine the precise orientation parameters by photogrammetric methods using an adapted aerial triangulation. Due to the different points of view towards the object the traditional aerotriangulation matching tools fail, as they produce a bunch of blunders and require a lot of manual work to achieve a sufficient solution. In this paper some approaches are discussed and results are presented for the most promising approaches. We describe a single step approach with an aerotriangulation using all available images; a two step approach with an aerotriangulation only of the vertical images plus a mathematical transformation of the oblique images using the oblique cameras excentricity; and finally the extended functional model for a bundle block adjustment considering the mechanical connection between vertical and oblique images. Beside accuracy also other aspects like efficiency and required manual work have to be considered.
\end{abstract}

\section{INTRODUCTION}

\subsection{Oblique Images}

For several purposes oblique camera systems become widely distributed. A good overview on the available systems is given by Petrie (2009). Oblique images fill the gap between vertical aerial images and terrestrial applications from stationary or mobile platforms. But whereas the hardware is developing fast, the required processes and software need extra effort.

The usage of oblique aerial imageries has some special challenges. The images taken from different points of view show the situation in quite different manner, especially the three dimensional objects in an urban environment. An other practical problem is to maintain an overview over the available images. Due to the trapeze footprints it is difficult to estimate the coverage of the single images, and to quantify the ground resolution and the overlap.

For many application high quality orientation data are essential. The calculation of the exterior orientation is the essence of this paper.

\subsection{Challenges for the Orientation of Oblique Imagery}

Automatic image matching tools designed for vertical images fail and produce a lot of blunders, when they are applied on oblique images. Tools designed for close range applications collapse due to the enormous number and size of the images used in mapping projects.

The BSF Swissphoto has developed an environment to handle and manage the images and the meta data of large aerial oblique imaging projects of several thousand images.

\subsection{AOS Sensor System}

The paper describes different strategies for the orientation of aerial oblique images. At BSF Swissphoto we operate the Aerial Oblique System (AOS). Details are described e.g. in Wiedemann et al., (2011). The system consists of three Trimble Rollei AIC 39 MPix cameras, build in a rotating sensor head in a stabilized mount with synchronized triggering by the flight management system IGI CCNS4. In bearing 1 one camera looks forward, one backward and the last straight down, in bearing 2 one camera looks left, one right and the last again downwards. With sufficient image sequences and overlap in the flight plan we can guarantee two vertical images and images from four different cardinal points to each object. In fact the overlap is much higher, many object points can be seen in $15-20$ different images (see also Fig 2).

Many applications of oblique images, like 3D city model texturing, visualization of navigation solutions or feature extraction require high accuracy interior and exterior orientation parameters. In many cases the quality of the direct sensor orientation is not sufficient, in particular at the outer edges of oblique images, where the influence of minor angle deviations have an increased influence.

The system is mechanical connected with DGPS/IMU system IGI Aerocontrol IId, also placed in the stabilized mount, following the movement of the mount, but not the rotation of the sensor head.

In a calibration field we do a boresight calibration for three cameras in both rotation bearings (along track and cross track). With this data we can calculate a set of initial exterior orientation data for each image by direct sensor orientation. 
Though the IGI Aerocontrol IId has a nominal accuracy of $\pm 0.004^{\circ}$ (Phi/Omega RMS) in practice we achieved an accuracy which is not sufficient for automatic facade texturing. So we realized the requirement of a more precise sensor orientation.

\subsection{User Requirements}

In a nutshell the following processes need orientation data with the accuracy of a single pixel in the object room. For a typical flight in $700 \mathrm{~m}$ above ground level an AOS pixel in the nadir is $10 \mathrm{~cm}$, in the center of an oblique images it has a size of about $15 \mathrm{~cm}$ on the ground. With an air speed of $120 \mathrm{kts}\left(60 \mathrm{~ms}^{-1}\right)$ this causes a forward motion of $6 \mathrm{~cm}$ with an exposure time of $1000 \mathrm{~s}^{-1}(0,4-0,7$ pixel).

In vertical images the errors of the $\mathrm{X}, \mathrm{Y}$ and $\mathrm{Z}$ and the rotation angles cause only linear dependent errors on the ground, whereas in oblique images error propagation has to be used.

If we estimate the GPS/INS accuracy with the following figures: $\pm 10 \mathrm{~cm}$ in $\mathrm{X}$ and $\mathrm{Y}, \pm 20 \mathrm{~cm}$ in $\mathrm{Z}, \pm 0.004^{\circ}$ in $\phi$ in and $\omega$ and $\pm 0.010^{\circ}$ in $\kappa$ (IGI 2012), we can estimate a total planar error of $\pm 25 \mathrm{~cm}$ on the ground caused by direct sensor orientation. In praxis this value proofed to be too optimistic (Grenzdörffer et al, 2008).

For some applications, like the monoscopic measurement (Höhle 2008) the requirements are lower. In this cases the direct sensor orientation might be sufficient.

\section{ORIENTATION STRATEGIES}

\subsection{Image stitching}

There is a small overlapping area between the three connected images of an exposure event, that allow a stitching of this three images to a seamless image butterfly (Fig. 1). The first idea was to perform an image stitching based on the overlapping zone between vertical and oblique images for introducing these

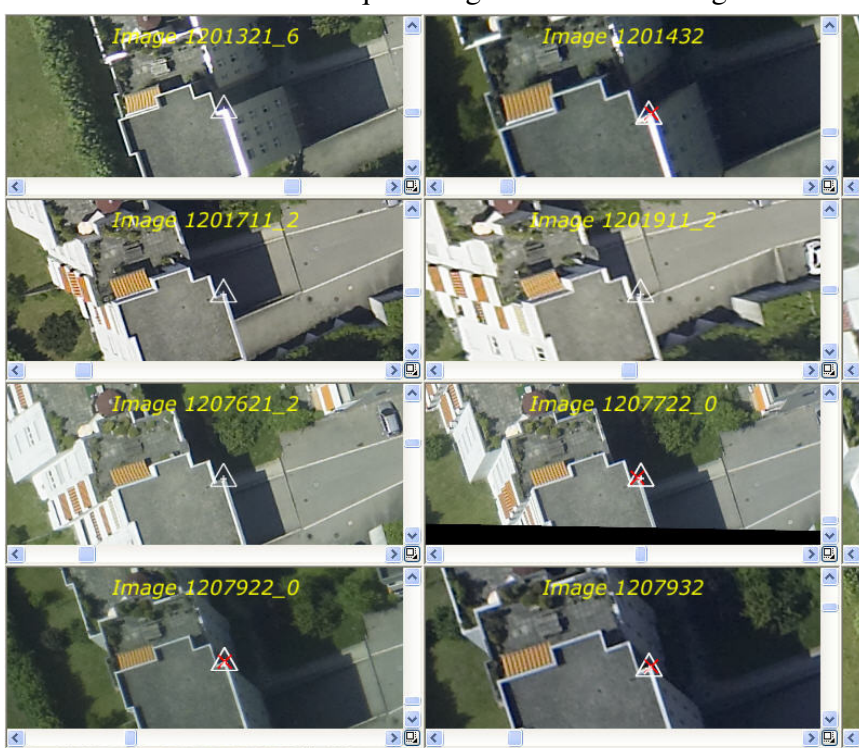

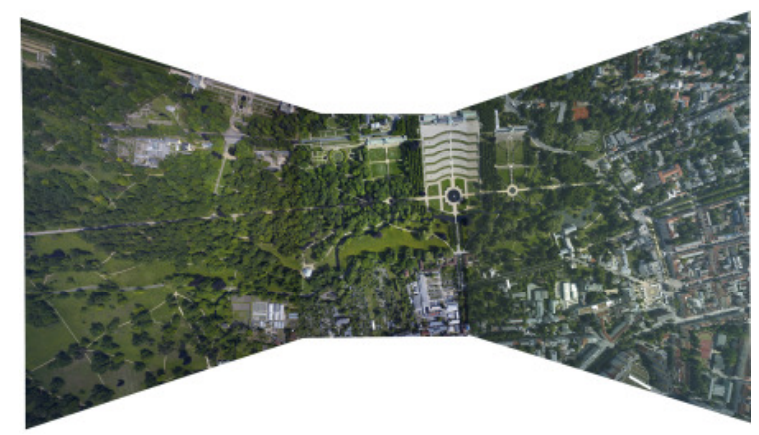

Figure 1: Stitched image triple

synthetic images into a standard aerotriangulation. This idea has been discarded due to the small overlapping zone between the images and other accuracy and practical issues. An increase of the overlapping zone would cause a serious loss of coverage.

\subsection{Single Step Approach}

The next approach was an aerotriangualtion over all images with the commercial triangulation software Inpho Match-AT or LPS (Jacobsen 2008). Due to very different aspect views of the situation, large scale differences and different illumination a lot of erroneous matching results caused weak or bogus tie points. overstraining the automatic blunder detection. The results assume to be good but are defective in some weak areas. That was the reason to search a better strategy.

\begin{tabular}{|l|r|r|r|}
\hline & $\phi$ [grad] & $\omega$ [grad] & $\kappa$ [grad] \\
\hline Camera 1 & 0,226 & 41,007 & 200,124 \\
& $\pm 0,052$ & $\pm 0,045$ & $\pm 0,042$ \\
\hline Camera 2 & 0,018 & $-40,739$ & 200,193 \\
& $\pm 0,054$ & $\pm 0,050$ & $\pm 0,044$ \\
\hline
\end{tabular}

Table 1: Relative orientation of oblique images versus vertical image (camera 0 ) based on an single step orientation in a test bed

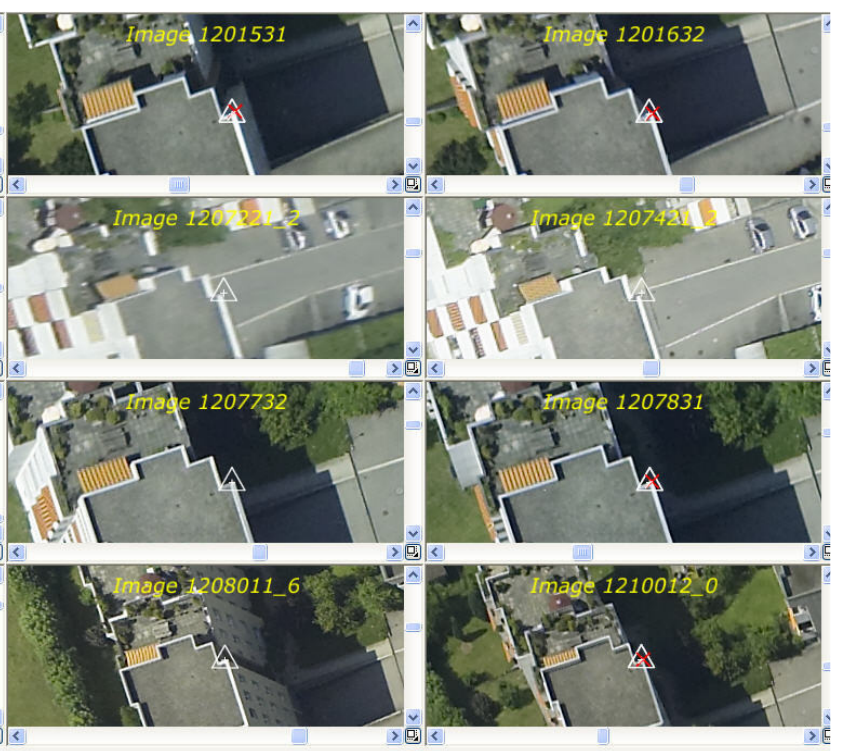

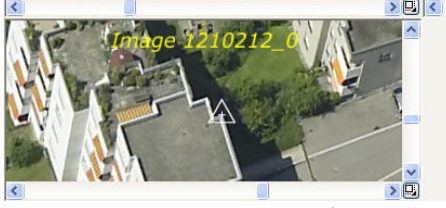

Figure 2: Manual Measurement of Tie Points in 17 Oblique Images with Match AT 
In smaller areas used as test bed, we realized the single step approach by performing an automatic tie point matching, followed by automatic and manual blunder elimination and additional interactive measurements (Figure 2). The results of the angle differences have been used to calculate the angle corrections and are shown in table 1.

\subsection{Two Step Approach}

Based on this experiences we changed the strategy toward an aerotriangualtion using only the vertical images. For this purpose the common software (e.g. Match-AT) is designed and performs very good, as proofed by additional checks using the triangulation software Bingo. The condition, allowing the use this approach, is a stable mechanical connection between the three cameras. The orientation of the two oblique cameras can be done by applying the offsets and rotation angles between vertical and oblique images to calculate the orientation data of the oblique images (table 1 and table 2). This step was realized by our own software.

\begin{tabular}{|l|r|r|r|}
\hline & $\Delta \mathrm{X}$ & $\Delta \mathrm{Y}$ & $\Delta \mathrm{Z}$ \\
\hline Camera 1 & $11,6 \mathrm{~cm}$ & $-9,6 \mathrm{~cm}$ & $-2,0 \mathrm{~cm}$ \\
\hline Camera 2 & $11,6 \mathrm{~cm}$ & $12,5 \mathrm{~cm}$ & $-2,0 \mathrm{~cm}$ \\
\hline
\end{tabular}

Table 2: Metric excentricity in camera centers measured at the sensor

The achieved geometric accuracy at the sensor is transformed into object space and compared with the accuracy achieved by direct sensor orientation and the aerotriangualtion of all images. The figures in chapter 3 show results of an orientation process with the two step approach.

Minor residual errors in the orientation of the vertical images, which can usually be ignored, may cause sometimes serious errors in the orientation of the oblique images due to extrapolation. Therefore the triangulation results of the vertical images have to be analysed very carefully.

\subsection{Extended Mathematical Model}

To reduce this problems, an extension of the bundle block approach for the extension of the mathematical model by introducing conditions of relative camera orientations is under development.

The traditional collinearity equations for aerial images express the image coordinates as functions (e.g. Albertz \&Wiggenhagen 2008) of object point coordinates and the orientation parameters. If we want to describe the exterior orientation parameters of camera 1 (oblique) we express this orientation parameters as a combination of the orientation parameters of camera 0 (vertical) image and the excentricity. The excentricity relative to the camera 0 is formulated by introducing 6 additional parameters describing the relative orientation of camera 1 (or 2) compared to camera 0.

$$
\begin{aligned}
& \omega_{1}=\omega_{0}+\Delta \omega_{1} \\
& \varphi_{1}=\varphi_{0}+\Delta \varphi_{1} \\
& \kappa_{1}=\kappa_{0}+\Delta \kappa_{1} \\
& X_{1}=X_{0}+E x z_{1} \cdot \tan \left(\kappa_{0}+\Delta \kappa_{E x 1}\right)
\end{aligned}
$$

$$
\begin{aligned}
& Y_{1}=Y_{0}+E x z_{1} \cdot \tan \left(\kappa_{0}+\frac{\pi}{2}+\Delta \kappa_{E x 1}\right) \\
& Z_{1}=Z_{0}+\Delta Z
\end{aligned}
$$

where

$$
\begin{aligned}
& \mathrm{Exz}_{1}=\begin{array}{l}
\text { horizontal, linear distance between } \\
\text { projection centers }
\end{array} \\
& \Delta \kappa_{\mathrm{Ex} 1}=\begin{array}{l}
\text { fixed rotation between camera } 0 \text { axis and } \\
\text { position of projection center of camera } 1
\end{array} \\
& \Delta \mathrm{Z}_{1}=\begin{array}{l}
\text { vertical difference between camera } 0 \text { and } \\
\text { camera } 1
\end{array} \\
& \Delta \omega_{1}, \Delta \phi_{1}, \Delta \kappa_{1} \text { difference between angles. }
\end{aligned}
$$

They are constant for all images of a mission. This mathematical model is simpler than the model with 6 transformation parameters and better suited for linearization, but supposes that the system remain in a horizontal position. This can be assumed by to the use of a stabilized mount with the AOS system.

This approach reduces the number of unknowns significantly. If we use the regular single step approach we need to evaluate the exterior orientation of 20 exposure with 3 cameras we would have to determine $20 \times 3 \times 6=360$ unknowns. The extended mathematical model would only need 120 orientation parameters for camera 0 plus $2 \times 6$ excentricity parameters, in sum only 132 unknowns.

The next step is to formulate the design matrix components and to realize a bundle block adjustment to determine this excentricity parameters and to compare them with the numerically determined values derived from the single and two step approaches.

\subsection{Orientation Based On DTM and Orthophotos}

Oblique images (Figure 3) give a general different aspect to the object than vertical images (Figure 4). Therefore image based correlation located in the image space is problematic. In some cases the difficulties are increased by different seasons (vegetation state), different radiometric characteristics of cameras and so on.

Using the a-priory knowledge provided by Digital Terrain Models (DTM), Digital Surface Models (DSM, Figure 5) and Digital Orthophotos (DOP) from other sources the matching strategies can be improved, by concentrating the tie point measurement to well suited areas of the images. An object space based approach might be useful and is under development.

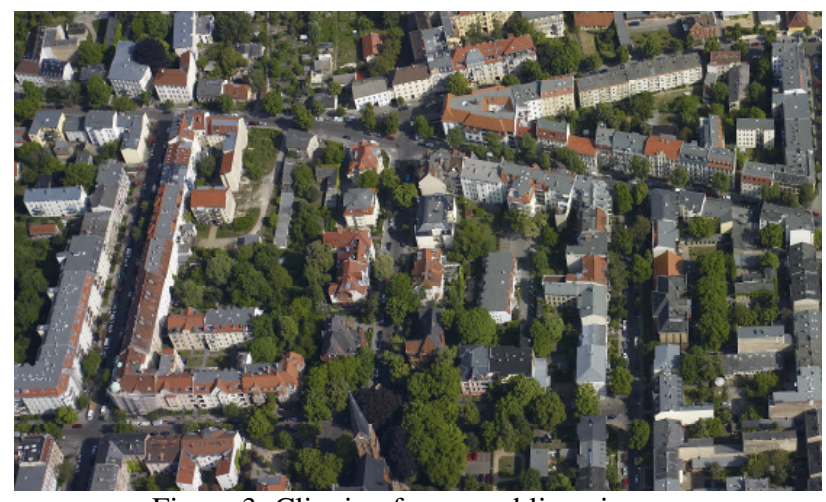

Figure 3: Clipping from an oblique image. 


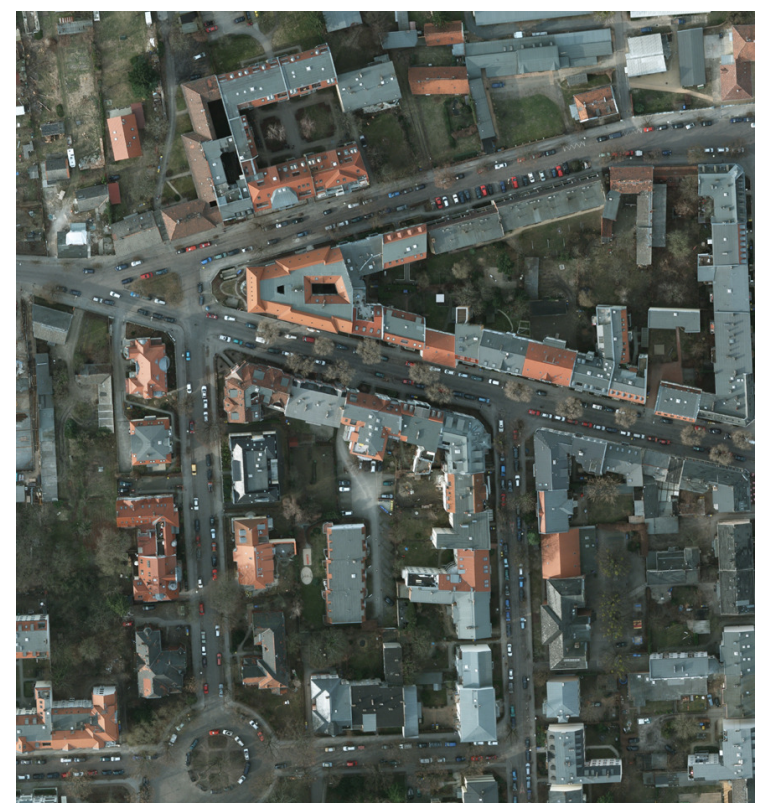

Figure 4: Digital Ortho Photo DOP based on Ultracam images of the orientation test bed

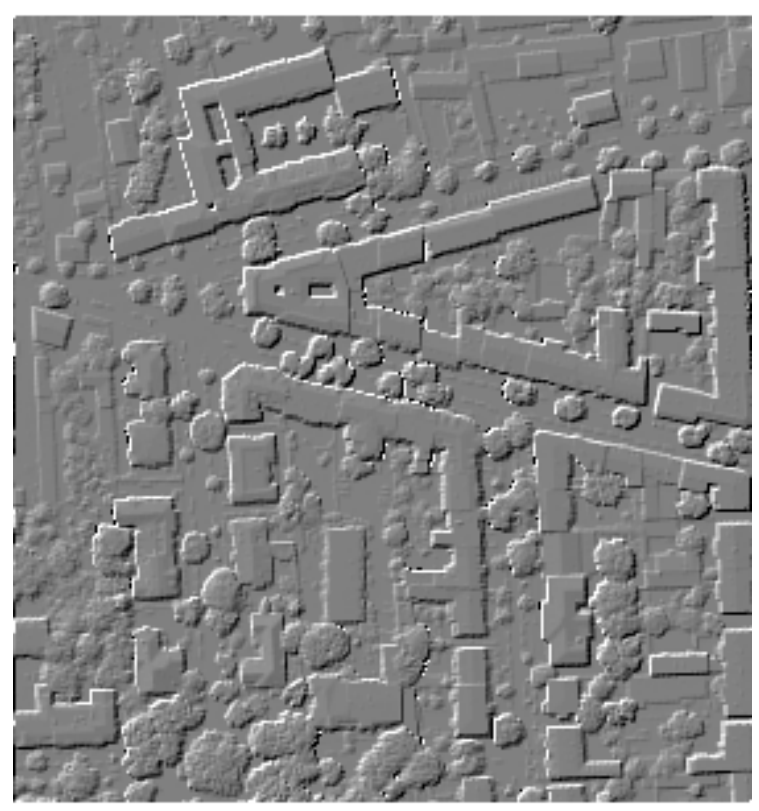

Figure 5: Digital Surface Model (DSM) of the test bed

\section{QUALITY CONTROL}

As mentioned above the quality assurance of the orientation data is essential to use the orientation data in further processes like texture mapping for 3D city models. In praxis some blunders were not detected by automatic processes, so we introduced quality assurance procedure based on images and existing vector data.

Projection of existing 3D vector data in the images, image chips of GCPs, digital othophotos derived from the oblique images (Figure 6), and projection of existing 3D building models into the original oblique images (Figure 7) are used for quality assurance processes. This proofs the operational applicability the selected two step approach.

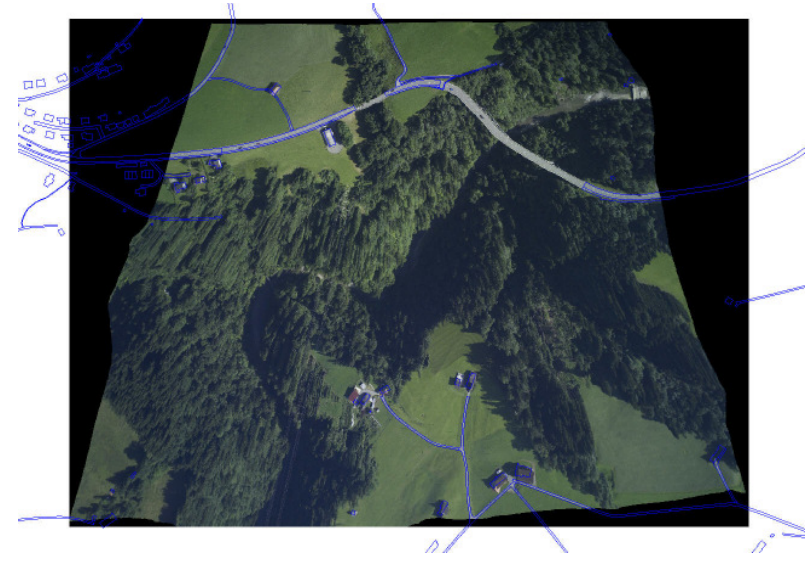

Figure 6: Quality check form of a differential rectified oblique image, superimposed by topographic features (e.g. Appenzell)

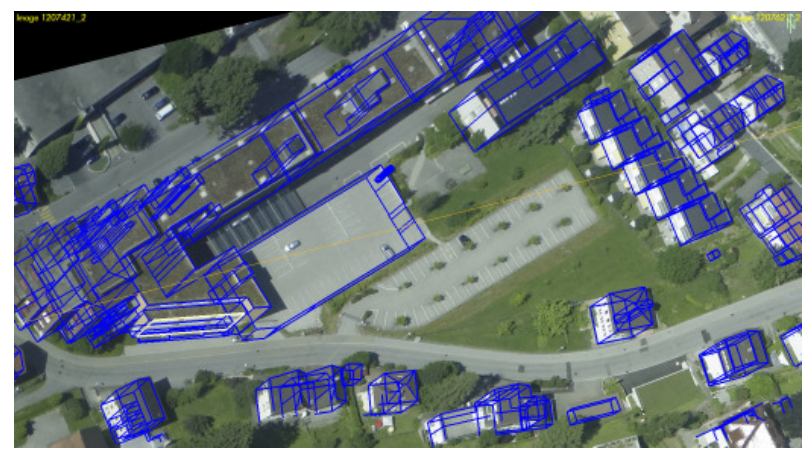

Figure 7: Quality check by superimposition of 3D building models into original oblique images

\section{CONCLUSIONS}

With the two step approach there is an operational approach to get sufficient accurate orientation data for most applications. But this approach still need a serious amount of manual work, especially during quality assurance. Therefore new approaches have to be defined using situation knowledge for the matching process and an extended mathematical model for the bundle block adjustment.

\section{ACKNOWLEGEMENTS}

The project underlying this report was funded by the German "Bundesminsiterium für Bildung und Forschung" under support code 03WKP21D. Responsible for the contend are the authors. The authors also want to thank all colleagues contributing to the work around the AOS projects. Thanks also to the Kanton Appenzell and Mr. Eugster from GEOINFO AG for the example data. 
International Archives of the Photogrammetry, Remote Sensing and Spatial Information Sciences, Volume XXXIX-B1, 2012

XXII ISPRS Congress, 25 August - 01 September 2012, Melbourne, Australia

SPONSORED BY THE



\section{REFERENCES}

Albertz, J. \& Wiggenhagen, M., 2008. Taschenbuch für Photogrammtrie und Fernerkundung - Guide for Photogrammetry and Remote Sensing. Wichmann, Karlsruhe, 2008

Grenzdörfer, G.J., Guretzki, M. \& Friedlander, I, 2008. Photogrammetric Image Acquisition and Image Analysis of Oblique Images - A New Challenge for the Digital Airborne System PFIFF. In: Photogrammetric Record 2008, Nr 12/2008 pp. 372-386.

Höhle, J., 2008: Photogrammetric Measurements in Oblique Aerial Images. In: Photogrammetrie Fernerkundung Geoinformation 2008, Nr 1/2008, pp. 7-14.

Jacobsen, K., 2008: Geometry of vertical and oblique image combinations: 28th EARSeL Symposium. Istanbul, 2008, $8 \mathrm{~S}$

Petrie, G., 2009: Systematic Aerial Oblique Photography Using Multiple Digital Cameras. PE\&RS, pp. 102-107.

Wiedemann, A., Moré, J., Möller, M., Wolf, R., 2011: Usage of Innovative Aerial Sensors for Conservation Purposes. 23 ${ }^{\text {rd }}$ CIPA Symposium, Prague 2011

References from websites: IGI Technical Specification Aerocontrol II http://www.igi.eu/aerocontrol.html (12 April 2012). 